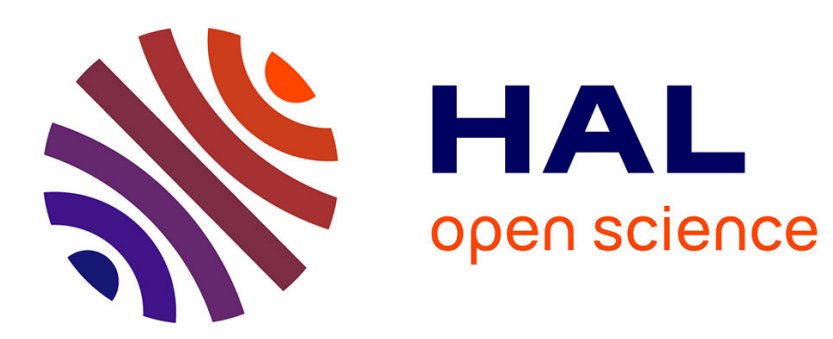

\title{
Hartwick's rule and maximin paths when the exhaustible resource has an amenity value
}

\author{
Antoine d'Autume, Katheline Schubert
}

\section{To cite this version:}

Antoine d'Autume, Katheline Schubert. Hartwick's rule and maximin paths when the exhaustible resource has an amenity value. 2008. halshs-00275765

\section{HAL Id: halshs-00275765 https://shs.hal.science/halshs-00275765}

Submitted on 25 Apr 2008

HAL is a multi-disciplinary open access archive for the deposit and dissemination of scientific research documents, whether they are published or not. The documents may come from teaching and research institutions in France or abroad, or from public or private research centers.
L'archive ouverte pluridisciplinaire HAL, est destinée au dépôt et à la diffusion de documents scientifiques de niveau recherche, publiés ou non, émanant des établissements d'enseignement et de recherche français ou étrangers, des laboratoires publics ou privés. 


\section{Documents de Travail du Centre d'Economie de la Sorbonne}
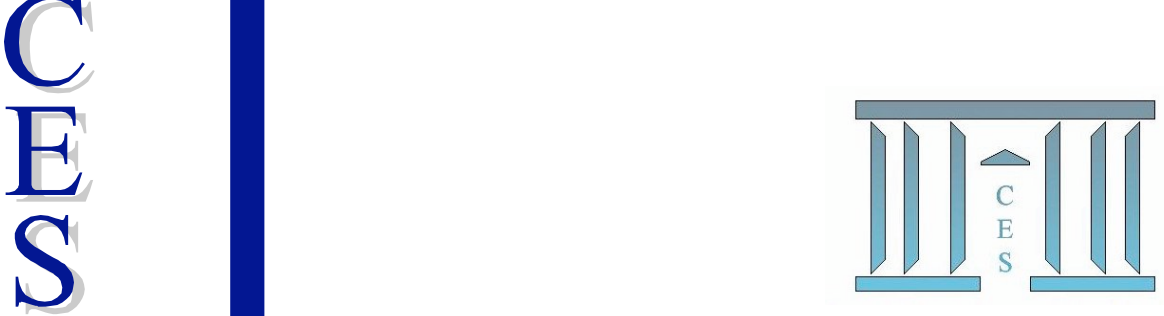

Hartwick's rule and maximin paths when the exhaustible resource has an amenity value

Antoine d'AuTUME, Katheline SCHUBERT

2008.31 


\title{
Hartwick's rule and maximin paths when the exhaustible resource has an amenity value
}

\author{
Antoine d'Autume and Katheline Schubert \\ Paris School of Economics and Université Paris 1 Panthéon-Sorbonne
}

April 14, 2008

\begin{abstract}
This paper studies the maximin paths of the canonical DasguptaHeal-Solow model when the stock of natural capital is a direct argument of well-being, besides consumption. Hartwick's rule then appears as an efficient tool to characterize solutions in a variety of settings. We start with the case without technical progress. We obtain an explicit solution of the maximin problem in the case where production and utility are Cobb-Douglas. When the utility function is CES with a low elasticity of substitution between consumption and natural capital, we show that it is optimal to preserve forever a critical level of natural capital, determined endogeneously. We then study how technical progress affects the optimal maximin paths, in the Cobb-Douglas utility case. On the long run path of the economy capital, production and consumption grow at a common constant rate, while the resource stock decreases at a constant rate and is therefore completely depleted in the very long run. A higher amenity value of the resource stock leads to faster economic growth, but to a lower long run rate of depletion. We then develop a complete analysis of the dynamics of the maximin problem when the sole source of well-being is consumption, and provide a numerical resolution of the model with resource amenity. The economy consumes, produces and invests less in the short run if the resource has an amenity value than if it doesn't, whereas it is the contrary in the medium and long runs. However, and without surprise, the resource stock remains for ever higher with resource amenity than without.
\end{abstract}

Keywords: exhaustible resources, sustainability, Hartwick's rule JEL classification: D9, Q01, Q3 


\section{Introduction ${ }^{1}$}

Sustainability requires, broadly speaking, to maintain possibilities of wellbeing for future generations. But there is no real consensus on a more precise definition, and sustainability can be interpreted in many different manners.

In order to gain insights on the sustainability issue, the literature has mainly proceeded by studying various versions of the canonical DasguptaHeal-Solow model (Dasgupta and Heal [1974], Solow [1974]), before resorting to more general set-ups. This model features a very simple economy using, besides man-made capital, the services of an exhaustible natural capital to produce its consumption good. The objective is to determine the intertemporal paths of depletion of natural capital and of accumulation of man-made capital, and whether natural capital must be entirely exhausted or not.

A first central issue is the one of substitutability between man-made capital and the exhaustible resource in production. The sustainability question is relevant only if this substitutability is poor, that is to say if the resource is essential in production (in the sense of Dasgupta and Heal [1974]). This is the case in the Cobb-Douglas case which we consider in this paper.

Natural capital, besides providing productive services, has an amenity value for consumers. Krautkraemer [1985] is one of the rare articles to examine a model where an exhaustible resource has an amenity value. An interpretation is to consider this resource to be natural capital, as its depletion appears to some extent irreversible. We follow this direction.

A second central issue, then, is the substitutability between consumption and the stock of natural capital in well-being. Weak sustainability admits some substitutability between consumption and the satisfaction derived from access to natural capital. To the contrary, strong sustainability implicitely considers this substitutability to be nil, and natural capital as the sole source of well-being.

A last central issue is the choice of the criterion of intertemporal welfare allowing to implement sustainability in the optimal growth model. Many agree to say that the usual discounted utilitarian criterion does not fit, as the process of discounting favors present generations at the expense of future ones. Other criteria have been proposed: undiscounted utilitarian criterion, maximin, Chichilnisky criterion. Each one has its drawbacks, and none of them is widely recognized as the good one.

We focus here on the maximin criterion. It produces paths sustainable

\footnotetext{
${ }^{1}$ We thank R. D. Cairns, N. V. Long, C. Withagen, participants at Paris 1 and Toulouse seminars, and two referees for useful comments, and especially M. Fleurbaey and C. Le Van for the proof in Appendix 1.
} 
in the sense that well-being is held constant along them. The adoption of a maximin criterion in an intertemporal setting has been initiated by Solow [1974]. He studies the Dasgupta and Heal [1974] model in the case where the social objective is to find an equitable growth path. Equity is taken in the sense that the consumption of the least well-off generation is maximized. Solow confesses to be "plus rawlsien que le Rawls", as Rawls himself was very reluctant to use a maximin welfare criterion for intertemporal problems (Rawls [1972]). Surprisingly, whereas the Solow model where utility only depends on consumption has been thoroughly studied in the literature, it has not been the case for the same model with natural capital as a direct argument of utility. Yet is seems natural as far as sustainability is concerned to express somehow that natural capital is a source of well-being besides consumption, and that both are not necessarily very substitutable. The first objective of this paper is to fill this gap in the literature.

From a methodogical point of view, we use a simple method to solve intertemporal maximin problems, which is simply to consider them as the limit case of a zero intertemporal elasticity of substitution. Moreover, we make a systematic use of Hartwick's rule (Hartwick [1977]) which states that the optimal investment rule, in a maximin context, is to conserve the total (man-made plus natural) value of capital for future generations. This allows us to obtain simple solutions, which has not much been done in the literature.

We obtain an explicit solution of the maximin problem in the DasguptaHeal-Solow framework, with a Cobb-Douglas production function, when the utility function is also Cobb-Douglas. We compute the optimal sustainable utility level and the paths of consumption, capital accumulation and resource depletion. Cobb-Douglas utility means a fairly high substitutability in preferences between consumption and the stock of resource, in the sense that it allows to sustain a positive utility level even though the stock of resource tends to zero. We then turn to the CES utility case, when substitutability in preferences between consumption and natural capital is lower. It is then necessary to preserve forever a critical level of natural capital, which will be determined endogeneously.

Then we study how technical progress affects the optimal maximin paths, in the Cobb-Douglas utility case. Contrary to Asheim et al. [2007], we make the usual assumption of an exponential technical progress. We give a complete characterization of the long run path of the economy, when both consumption and the stock of resource are arguments of utility. We show that capital, production and consumption grow at a common constant rate, related in a specific way to the rate of technical progress, while the resource stock decreases at another constant rate and is completely depleted in the long run. However, the higher the amenity value of the resource stock, the 
lower the long run rate of depletion. On the other hand, the physical rate of growth is an increasing function of the amenity value. Faster growth does not mean faster resource depletion.

We then build on a result derived by Cairns and Long [2006] to develop a complete analysis of the dynamics of the maximin problem when the sole source of well-being is consumption. Unfortunately, this method does not apply in the general case of resource amenity and we have to resort to a numerical resolution of the model. The simulations show that initially the economy consumes, produces and invests less if the resource has an amenity value than if it doesn't, which allows slower depletion of the resource stock. Then, as this stock decreases, the economy must increase its reliance on physical production. Capital accumulation, with the help of technological progress, allows the economy to maintain a high rate of growth, without depleting too quickly the resource stock. The resource stock remains for ever higher than in the case of no resource amenity.

The remainder of the paper is organized as follows. Section 2 presents the method we propose to solve intertemporal maximin problems. Section 3 studies the maximin paths of a Dasgupta-Heal-Solow economy in which the resource stock has an amenity value. We introduce in section 4 technical progress and study how it modifies the optimal maximin paths. Section 5 concludes.

\section{How to solve intertemporal maximin prob- lems}

Several methods are available to solve intertemporal maximin programs.

The problem is to maximize

$$
\min _{t} u_{t}
$$

under technical constraints describing capital accumulation and resource depletion. $\quad u_{t}$ denotes the utility level at time $t$, which depends on various variables.

Léonard and Long [1992] state the objective as maximizing

$$
W=\int_{0}^{\infty} \rho e^{-\rho t} u d t=u,
$$

for an arbitrary discount factor $\rho>0$, and add the following condition:

$$
u_{t} \geq u \quad \forall t
$$


to the technical constraints.

Cairns and Long [2006] depart from this approach by directly maximizing the sustained level of utility $u$, and solving a somewhat unusual control problem. They provide a general and rigorous analysis of this kind of maximin problems.

We propose here another method based on the simple observation that the limit of a CES function when the elasticity of substitution between the variables tends to zero is in fact the min function. A justification is given in Appendix 1.

We solve a usual discounted utilitarian program:

$$
\max W=\int_{0}^{\infty} e^{-\rho t} \frac{u_{t}^{1-1 / \sigma}}{1-1 / \sigma} d t
$$

for an arbitrary discount rate $\rho$ and an arbitrary intertemporal elasticity of substitution $\sigma$. We then let $\sigma$ tend towards zero in the solution in order to obtain the maximin path. In our setting, this yields a solution with a constant utility level, that is a regular maximin $\mathrm{path}^{2}$. We admittedly face a theoretical issue regarding the validity of this limit method. We do not solve the general issue but it is easy to check that we get the same optimality conditions as those which follow from the first two methods.

The difference in solution methods is not only technical however. The first two methods model equity by way of a specific constraint in an optimization problem. Cairns and Long [2006] indeed stress the role of the Lagrange multiplier associated to this constraint, which they interpret as the shadow price of equity. They proceed to derive interesting properties of this shadow price, which play the role of virtual discount factors.

Our method only assumes that society is reluctant to substitute wellbeing across dates. No shadow price of equity emerges in our method, even if it is of course possible to relate the different methods, as we do in Appendix 2 .

\section{Maximin paths when the resource stock has an amenity value}

Whereas optimal growth models dealing with pollution always take into account the desutility generated by its stock, models dealing with exhaustible resources almost never introduce the stock not yet extracted as an argument

\footnotetext{
${ }^{2}$ See Dixit et al. [1980] as well as Buchholz et al. [2005] for a discussion of regular paths.
} 
of well-being. This omission may seem rather natural if exhaustible resources are defined in a narrow way as fossil fuels or mineral ores. Yet, extraction and use of fossil fuels contribute to global warming and therefore have a negative amenity value. Stollery [1998] thus studies the influence of global warming on utility as well as production possibilities. Moreover, the resource stock can be more broadly interpreted as natural capital, which certainly has an amenity value, and the depletion of which is largely irreversible.

We therefore study the maximin paths of the canonical Dasgupta-HealSolow model with the stock of resource as an argument of utility, besides consumption. Krautkraemer [1985] studies the same model with a discounted utilitarian social welfare function. Stollery [1998] considers a maximin problem where temperature is inversely linked to the remaining stock of exhaustible resource. Temperature appears as an argument both in utility and production functions. He is only able, however, to obtain a closed-form solution for the case where temperature only affects production. To the best of our knowledge, the maximin paths have not been calculated yet in a more general framework.

\subsection{The model}

We take as departure point the utilitarian central planner's program:

$$
\max \int_{0}^{\infty} e^{-\rho t} U\left(c_{t}, X_{t}\right) d t
$$

under the usual technical constraints

$$
\begin{gathered}
\dot{K}_{t}=F\left(K_{t}, x_{t}\right)-c_{t}, \\
\dot{X}_{t}=-x_{t},
\end{gathered}
$$

and the initial conditions

$$
K_{0} \text { and } X_{0} \text { given. }
$$

Equation (6) describes capital accumulation. Production $Y_{t}=F\left(K_{t}, x_{t}\right)$ is a function of the capital stock $K_{t}$ and of the flow of exhaustible resource extracted at time $t, x_{t} . X_{t}$ is the stock of exhaustible resource at time $t$, depleted by extraction (equation (7)).

We assume that

$$
U\left(c_{t}, X_{t}\right)=\frac{u\left(c_{t}, X_{t}\right)^{1-1 / \sigma}}{1-1 / \sigma},
$$

with a given $u(c, X)$ function. 
The first order necessary conditions are (dropping the time index):

$$
\begin{aligned}
U_{c} & =\lambda \\
F_{x} & =q \\
\frac{\dot{\lambda}}{\lambda} & =\rho-F_{K} \\
\frac{\dot{q}}{q}+\frac{U_{X} / U_{c}}{q} & =F_{K}
\end{aligned}
$$

where $\lambda$ is the shadow price of capital and $q$ the relative shadow price of the resource stock in terms of capital. Equation (13) is a modified version of the Hotelling's rule: the return of the resource now includes its relative amenity value.

As $U_{c}=u^{-1 / \sigma} u_{c}$, we obtain

$$
-\frac{1}{\sigma} \frac{\dot{u}}{u}+\frac{\dot{u}_{c}}{u_{c}}=\rho-F_{K}
$$

or

$$
\frac{\dot{u}}{u}=\sigma\left(F_{K}+\frac{\dot{u}_{c}}{u_{c}}-\rho\right),
$$

which indeed reduces to the constancy of $u$ when $\sigma$ tends to zero.

The marginal rate of substitution between resource amenity and consumption is independent of $\sigma$, as $U_{X} / U_{c}=u_{X} / u_{c}$.

First order conditions for the maximin problem are therefore

$$
\begin{aligned}
F_{x} & =q \\
\frac{\dot{q}}{q}+\frac{u_{X} / u_{c}}{q} & =F_{K} \\
u=\text { constant } &
\end{aligned}
$$

to which we should add the technical constraints (6) and (7) giving the evolution of $K$ and $X$, and the initial conditions (8).

The discount rate $\rho$ disappears, as the weights attached to utilities at different dates become irrelevant when agents do not want to consider any substitution between them ${ }^{34}$.

\footnotetext{
${ }^{3}$ Indeed, the weights in a CES production function disappear when the elasticity of substitution tends to zero.

${ }^{4}$ In a more general setting, letting $\sigma$ tend to zero would leave supplementary optimal cross relationships, in addition to the constancy of $u$. These conditions are the ones which appear in Cairns and Long [2006].
} 


\subsection{Hartwick's rule}

A nice way to solve this system is to use Hartwick's rule (Hartwick [1977]). The original Hartwick's rule indeed holds in our model with the resource stock in the utility function. Its use simplifies the resolution and even allows an explicit solution in the Cobb-Douglas case.

In its simplest form, Hartwick's rule states that, if on an efficient path the total value of net investment, or genuine saving ${ }^{5}$, is zero at each point in time, then utility is constant. In other words a society which invests the rents obtained from the extraction of the exhaustible resource in net accumulation of man-made capital follows an equitable growth path. In the original paper by Hartwick, this rule was proved to hold for the Dasgupta-Heal-Solow model with utility depending only on consumption.

Dixit, Hammond and Hoel [1980] proved that a generalized version of the rule holds in a large class of models: utility is constant on an efficient path if the discounted value of total investment is constant (and not necessarily zero). They also prove the converse result, using additional assumptions that Withagen and Asheim [1998] are able to relax. Buchholz, Dasgupta and Mitra [2005] prove that in an exhaustible resource model à la Dasgupta-HealSolow with a general specification of the technology in which the resource is important, and utility depending only on consumption, the constant is indeed zero, so that the original Hartwick's rule holds. Finally, Cairns and Long [2006] generalize further Hartwick's rule.

Let us define the value of total investment as

$$
G=\dot{K}+q \dot{X}
$$

The Dixit, Hammond and Hoel [1980] generalized rule states that the discounted value $e^{-R} G$ of total investment (with $R_{t}=\int_{0}^{t} F_{K}\left(K_{s}, x_{s}\right) d s$ ) is constant on any competitive constant utility path. It remains to prove that $G=0$ along any feasible solution of the optimality conditions. We adapt the Buchholz, Dasgupta and Mitra [2005] proof in Appendix 3 to show that this is the case in our setting.

Thus:

$$
\dot{K}=-q \dot{X}=q x .
$$

Let us now consider the case of a Cobb-Douglas production function ${ }^{6}$ :

$$
Y=F(K, x)=K^{\alpha} x^{\beta}, \quad \alpha, \beta>0, \quad \alpha+\beta<1 .
$$

\footnotetext{
${ }^{5}$ The term "genuine saving" has been introduced by Hamilton and Clemens [1999].

${ }^{6}$ Labor implicitly appears as a third production factor and production takes place under constant returns to scale. We assume that the quantity of labor is an exogenous constant which is normalized to unity.
} 
Hartwick's rule implies in this case, using equation (15),

$$
\begin{aligned}
\dot{K} & =\beta Y, \\
c & =(1-\beta) Y .
\end{aligned}
$$

The saving rate is constant along the optimal path, and equal to the resource share in the production function $\beta$. Contrary to the Solow [1974] case where natural capital is not an argument of utility, and $c$ is constant along a maximin case, the production level will not be constant.

Inverting the utility function allows us to express consumption as a function $C(u, X)$ of the utility level $u$ and the resource stock $X$.

For a constant utility level $u$, the model reduces to the following system:

$$
\begin{aligned}
\dot{K} & =\beta Y \\
\dot{X} & =-Y^{1 / \beta} K^{-\alpha / \beta} \\
Y & =\frac{C(u, X)}{1-\beta} .
\end{aligned}
$$

Substituting $Y$ in the first two equations yields a differential system in $K$ and $X$. This system can be solved by time elimination and variable separation. The ratio of equations (24) and (23) yields ${ }^{7}$

$$
\frac{d X}{d K}=-\frac{1}{\beta}\left(\frac{C(u, X)}{1-\beta}\right)^{1 / \beta-1} K^{-\alpha / \beta} .
$$

This allows us to separate variables:

$$
-\left(\frac{C(u, X)}{1-\beta}\right)^{-\frac{1-\beta}{\beta}} d X=\frac{1}{\beta} K^{-\alpha / \beta} d K .
$$

Integration of equation (26) yields, after interchanging the bounds of the integral on the left-hand side as $X$ is smaller than $X_{0}$ :

$$
\int_{X}^{X_{0}}\left(\frac{C(u, \xi)}{1-\beta}\right)^{-\frac{1-\beta}{\beta}} d \xi=\frac{1}{\beta} \int_{K_{0}}^{K} \kappa^{-\alpha / \beta} d \kappa,
$$

or

$$
\Phi_{L}\left(X_{0}, u\right)-\Phi_{L}(X, u)=\Phi_{R}(K)-\Phi_{R}\left(K_{0}\right)
$$

where $\Phi_{L}(X, u)$ and $\Phi_{R}(K)=K^{1-\alpha / \beta} /(\beta-\alpha)$ are primitive functions of the integrands on the left and right hand sides, respectively.

\footnotetext{
${ }^{7}$ From Hartwick's rule, $\dot{X} / \dot{K}$ is simply $-1 / q$.
} 
Function $\Phi_{L}(X, u)$ is increasing in $X$ and the left-hand side positive. We assume $\beta<\alpha$. As we shall see, if the share of the resource in production is higher than the share of capital, no constant utility level is sustainable. When $\alpha>\beta, \Phi_{R}(K)$ is increasing in $K$ so that the right-hand side is also positive if $K$ is larger than $K_{0}$. It is self-evident that this must be the case, as the utility level can be maintained only if capital accumulation compensates for the depletion of the natural resource.

The right-hand side increases from 0 to $K_{0}^{1-\alpha / \beta} /(\alpha-\beta)$ when $K$ increases from $K_{0}$ to infinity.

The left-hand side is a decreasing function of $X$ and $u$, and an increasing function of $X_{0}$.

Equation (27) basically determines $X$ as a function of $K$ and $u$. It allows us to characterize sustainable paths, and the optimal one among them.

To go further, we study two cases: the case of a Cobb-Douglas utility function, representative of a high substitutability in preferences between consumption and natural capital, and the one of a CES utility function with a low elasticity of substitution between the two sources of well-being.

\subsection{A Cobb-Douglas utility function}

Let us first consider the case of a Cobb-Douglas utility function:

$$
u(c, X)=c X^{\varepsilon} \Leftrightarrow C(u, X)=u X^{-\varepsilon}, \quad \varepsilon \geq 0 .
$$

Then

$$
\Phi_{L}(X, u)=\left(\frac{u}{1-\beta}\right)^{-\frac{1-\beta}{\beta}} \frac{X^{\phi}}{\phi}
$$

with

$$
\phi=1+\frac{1-\beta}{\beta} \varepsilon .
$$

Equation (28) becomes

$$
\left(\frac{u}{1-\beta}\right)^{-\frac{1-\beta}{\beta}} \frac{X_{0}^{\phi}-X^{\phi}}{\phi}=\frac{K_{0}^{1-\alpha / \beta}-K^{1-\alpha / \beta}}{\alpha-\beta} .
$$

A diagrammatic analysis (figure 1) helps us to characterize the optimal path.

The N-W orthant of figure 1 plots function $\Phi_{L}\left(X_{0}, u\right)-\Phi_{L}(X, u)$ as a function of $X$, for various levels of $u$. In the Cobb-Douglas case, the function 


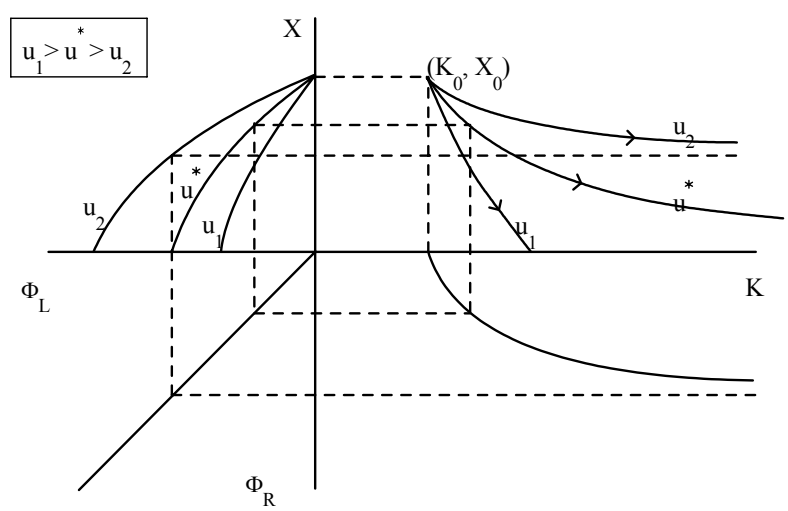

Figure 1: Sustainability in the Cobb-Douglas utility case

is defined on the interval $\left(0, X_{0}\right)$ and decreases from $\Phi_{L}\left(X_{0}, u\right)$ to zero. A higher value of $u$ implies lower values of $\Phi_{L}\left(X_{0}, u\right)-\Phi_{L}(X, u)$.

The S-E orthant plots function $\Phi_{R}(K)-\Phi_{R}\left(K_{0}\right)$. It increases from zero to a finite upperbound $-\Phi_{R}\left(K_{0}\right)$.

We use the $45^{\circ}$ line in the $\mathrm{S}-\mathrm{W}$ orthant to draw the resulting $X\left(K, u, X_{0}, K_{0}\right)$ curve in the N-E orthant. This curve describes the trajectory of the capital and resource stocks. The arrow depicts the direction followed from the initial point: the resource stock is decreasing, whereas the capital stock is increasing.

In the case of a constant utility level equal to $u_{1}$, the trajectory is not sustainable. The resource stock reaches zero in finite time.

In the case of a lower utility level $u_{2}$, the trajectory is sustainable. Capital tends to infinity. The limit level of the resource stock is strictly positive.

The optimal trajectory is associated with the highest sustainable utility level $u^{*}$. Capital tends to infinity and the resource stock tends to zero. The fact that the resource is essential in production and has an amenity value is not sufficient to prevent asymptotic depletion in the long run. Capital accumulation, on the production side, and increased consumption, on the welfare side, will compensate for the decrease of the resource stock.

Making $X=0$ and $K \rightarrow \infty$ in equation (28) then yields

$$
\Phi_{L}\left(X_{0}, u\right)=-\Phi_{R}\left(K_{0}\right)
$$

and provides, in the Cobb-Douglas utility case, the optimal utility level:

$$
u^{*}=(1-\beta)\left(\frac{\alpha-\beta}{\phi} K_{0}^{\frac{\alpha-\beta}{\beta}} X_{0}^{\phi}\right)^{\frac{\beta}{1-\beta}} \text {. }
$$


From $(28)$ and (31), the resulting optimal path in the $(K, X)$ plane is

$$
\Phi_{L}\left(X, u^{*}\right)=-\Phi_{R}(K)
$$

or

$$
u^{*}=(1-\beta)\left(\frac{\alpha-\beta}{\phi} K^{\frac{\alpha-\beta}{\beta}} X^{\phi}\right)^{\frac{\beta}{1-\beta}} .
$$

This last equation determines an aggregate of both stocks

$$
B \stackrel{\text { def }}{=} K^{\frac{\alpha-\beta}{\beta}} X^{\phi}=K_{0}^{\frac{\alpha-\beta}{\beta}} X_{0}^{\phi}
$$

which is conserved along the optimal path and determines the sustainable utility level.

In other words, equation (33) defines a family of iso-utility curves in the $(K, X)$ plane. Initial endowments $\left(K_{0}, X_{0}\right)$ and, more precisely, their aggregate determine the relevant curve and the associated sustainable utility level. The economy will then follow this iso-utility curve in a downward direction, as man-made capital substitutes for natural capital. From Hartwick's rule, the price vector $(1, q)$ is orthogonal at each point in time to vector $(\dot{K}, \dot{X})$, and therefore to the iso-utility curve.

These results generalize the Solow [1974] analysis of the case where the resource stock does not appear in the utility function. The Solow formula is recovered when $\varepsilon=0$ and $\phi=1$.

We may also use our diagram to analyze the case $\alpha<\beta$ of a high share of resource use in production. Function $\Phi_{R}(K)-\Phi_{R}\left(K_{0}\right)$ now increases from zero to infinity, as $K$ increases from $K_{0}$ to infinity. All trajectories correspond to the $u_{1}$ case of the previous diagram. No sustainable path exists.

We relegate in Appendix 4 the complete resolution of this problem. We show that the paths followed by the stocks of capital and resource are quasiarithmetic:

$$
\begin{aligned}
& K_{t}=\left[(1-\pi) A t+K_{0}^{1-\pi}\right]^{\frac{1}{1-\pi}} \\
& X_{t}=B^{1 / \phi}\left[(1-\pi) A t+K_{0}^{1-\pi}\right]^{-\frac{\alpha-\beta}{\beta+\varepsilon(1-\alpha)}}
\end{aligned}
$$

where

$$
\pi=\frac{\varepsilon(\alpha-\beta)}{\beta+\varepsilon(1-\beta)}, \quad 0<\pi<1,
$$

and $A$ and $B$ are constants depending on initial stocks.

The rate of growth of the physical capital stock decreases to zero as time tends to infinity. We know that consumption tends to infinity in order to 
preserve a constant utility level as the resource amenity tends to zero. As $C=$ $(1-\beta) Y$, production does the same. Yet $\dot{K} / K=\beta Y / K$ decreases to zero, which shows that capital grows faster than production and consumption. With Cobb-Douglas production, this also means that the real interest rate decreases to zero. As resource use decreases and capital accumulates, the marginal productivity of capital is bound to decrease.

The rate of depletion of the natural capital stock also decreases to zero. This means that the depletion of the natural stock is very slow. It would be easy to check that the half-life of the resource stock increases as time passes.

It is also worthwhile determining the shadow value of equity defined by Cairns and Long [2006]. We show in Appendix 4 that

$$
w_{t}=A \frac{\alpha-\beta}{\beta} K_{0}^{\frac{\alpha-\beta}{\beta}}\left((1-\pi) A t+K_{0}^{1-\pi}\right)^{-\frac{\alpha+\varepsilon(1-\alpha)}{\beta+\varepsilon(1-\alpha)}},
$$

which yields a decreasing (but not hyperbolic, as noticed by Cairns and Long [2006]) discount rate:

$$
\rho_{t}=-\frac{\dot{w}_{t}}{w_{t}}=\frac{\alpha+\varepsilon(1-\alpha)}{\beta+\varepsilon(1-\alpha)} \frac{(1-\pi) A}{(1-\pi) A t+K_{0}^{1-\pi}} .
$$

The shadow price of equity is model-dependent. There exists no general specification of the discount factor that would garantee that the optimal path in a discounted utilitarian problem is equitable.

\subsection{A CES utility function}

Let us now consider the case of a CES utility function:

$$
\begin{gathered}
u(c, X)=\left(\omega c^{\frac{\mu-1}{\mu}}+(1-\omega) X^{\frac{\mu-1}{\mu}}\right)^{\frac{\mu}{\mu-1}} \\
\Leftrightarrow C(u, X)=\left(\frac{1}{\omega}\left(u^{\frac{\mu-1}{\mu}}-(1-\omega) X^{\frac{\mu-1}{\mu}}\right)\right)^{\frac{\mu}{\mu-1}},
\end{gathered}
$$

where $\mu(>0, \neq 1)$ is the elasticity of substitution between the two arguments of utility and $\omega>0$ a share parameter.

If consumption and the stock of natural resource are poor substitutes in utility $(\mu<1)$, the case we are interested in, it is possible to reach a given utility level $u>0$ only if the natural resource stock is high enough:

$$
X>X_{\min }(u)=u(1-\omega)^{\frac{\mu}{1-\mu}} .
$$

Then, $C(u, X)$ tends towards infinity when $X$ decreases towards $X_{\min }(u)$. 


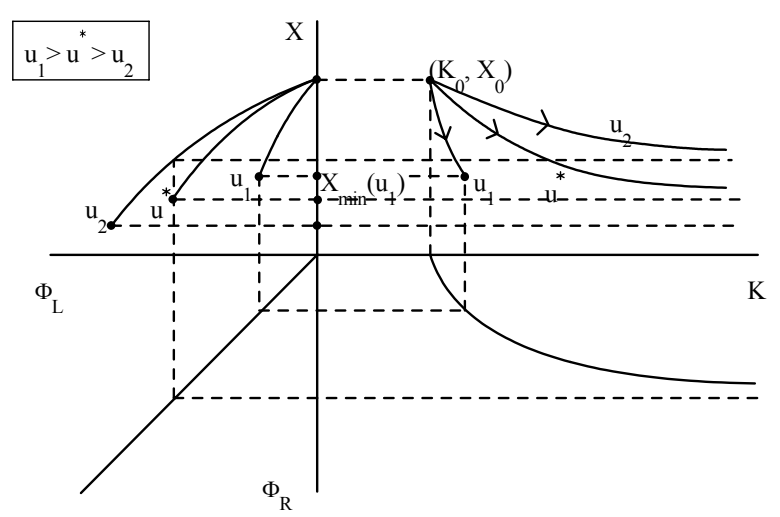

Figure 2: Sustainability in the CES utility case

Let

$$
g(u, X)=\left(\frac{C(u, X)}{1-\beta}\right)^{-\frac{1-\beta}{\beta}} .
$$

$g(u, X)$ is positive, and increases from 0 to $g\left(u, X_{0}\right)$ when $X$ increases from $X_{\min }(u)$ to $X_{0}$ (under the assumption that $u$ is low enough, so that $X_{\min }(u)<$ $\left.X_{0}\right)$.

Let again $\Phi_{L}(X, u)$ be a primitive of $g(u, X)$ and reconsider equation (28)

$$
\Phi_{L}\left(X_{0}, u\right)-\Phi_{L}(X, u)=\Phi_{R}(K)-\Phi_{R}\left(K_{0}\right)
$$

The left-hand side decreases from $\Phi_{L}\left(X_{0}, u\right)-\Phi_{L}\left(X_{\min }(u), u\right)$ to 0 when $X$ increases from $X_{\min }(u)$ to $X_{0}$.

With the same kind of arguments as in the Cobb-Douglas case, and the help of figure 2, it is easy to see that the maximal sustainable utility level is $u^{*}$ satisfying

$$
\Phi_{L}\left(X_{0}, u^{*}\right)-\Phi_{L}\left(X_{\min }\left(u^{*}\right), u^{*}\right)=-\Phi_{R}\left(K_{0}\right)
$$

Contrary to the Cobb-Douglas case, $u^{*}$ cannot be calculated explicitely in a simple form. But the important point is that when the utility function is CES, with a poor substitutability between consumption and natural capital, the equitable path does not lead to the exhaustion of the resource. With a Cobb-Douglas production function, it is impossible to produce without using up the resource. It is therefore impossible to maintain a constant stock of resource. But capital accumulation makes it possible to slow down resource 
depletion and, now, to preserve forever a critical level of natural capital. The resource stock tends in infinite time to an endogenous value $X_{\min }\left(u^{*}\right)$. Some form of strong sustainability is obtained. The advantage of this approach is that this critical natural capital is endogenously determined and depends on the parameter of preference $\mu$ and also, through the sustainable level of utility $u^{*}$, on the technological parameters $\alpha$ and $\beta$ and on the values of the initial stocks.

Equations (28), for $u=u^{*}$, and (45) yield

$$
\Phi_{L}\left(X, u^{*}\right)-\Phi_{L}\left(X_{\min }\left(u^{*}\right), u^{*}\right)=-\Phi_{R}(K)
$$

This implicitely defines the optimal path in the $(K, X)$ plane and, more generally, the set of iso-utility curves.

We cannot obtain explicit solutions for the trajectories but we can derive approximate solutions when times goes to infinity and thus characterize the long run evolution.

Consider (26) and (42), for given $u^{*}$. As time goes to infinity, $X$ tends to $X_{\min }\left(u^{*}\right)$ and $K$ to infinity. Then,

$$
\begin{gathered}
u^{* \frac{\mu-1}{\mu}}-(1-\omega) X^{\frac{\mu-1}{\mu}} \simeq(1-\omega) \frac{1-\mu}{\mu}\left(X_{\min }\right)^{-1 / \mu}\left(X-X_{\min }\right) \\
C(u, X) \simeq\left[\frac{1-\omega}{\omega} \frac{1-\mu}{\mu}\right]^{\frac{\mu}{\mu-1}}\left(X_{\min }\right)^{\frac{1}{1-\mu}}\left(X-X_{\min }\right)^{\frac{\mu}{\mu-1}}=\Psi\left(u^{*}\right)\left(X-X_{\min }\right)^{\frac{\mu}{\mu-1}}
\end{gathered}
$$

with a constant $\Psi\left(u^{*}\right)$. Let $\hat{X}=X-X_{\min }\left(u^{*}\right)$. An approximate long run differential equation is therefore

$$
\frac{d \hat{X}}{d K}=-\frac{1}{\beta}\left(\frac{\Psi\left(u^{*}\right) \hat{X}^{-\frac{\mu}{\mu-1}}}{1-\beta}\right)^{\frac{1-\beta}{\beta}} K^{-\alpha / \beta}
$$

This equation is identical to the one which holds in the Cobb-Douglas utility case, with $\Psi\left(u^{*}\right) \hat{X}^{-\frac{\mu}{\mu-1}}$ replacing $u^{*} X^{-\varepsilon}$. Thus the convergence of $X$ to $X_{\min }\left(u^{*}\right)$ is similar to the convergence of $X$ to zero in the Cobb-Douglas case.

\section{Technical progress and maximin paths}

We now examine how technical progress affects the depletion of the resource stock. Does it allow to maintain a positive stock of resource, even in the 
Cobb-Douglas utility case? Should the physical capital stock still increase without limits, or can it converge to a finite level or even to zero as Solow [1974] guessed, writing that "society asymptotically consumes its stock of capital as it consumes its pool of resource, relying on technical progress to maintain net output and consumption", but adding that "this is far from certain, because in a growing system, any constant capital stock becomes effectively small"? Surprisingly, a complete analysis is still lacking. Cairns and Long [2006] treat the maximin consumption case. They provide an original condition which supplements Hartwick's rule and simplifies the dynamic analysis. Unfortunately, this condition does not hold in the more general case of resource amenity. We develop a complete analysis of the maximin consumption case, which Cairns and Long [2006] only sketched. We proceed with a characterization of the long run situation in the (Cobb-Douglas) case of resource amenity and provide a numerical resolution of the model.

\subsection{The model}

Although the issue is immaterial in the Cobb-Douglas case, we start with a labor-augmenting technical progress at the constant rate $\gamma$ :

$$
Y=K^{\alpha} x^{\beta} A^{1-\alpha-\beta}, \quad \dot{A} / A=\gamma, \quad A_{0}=1 .
$$

The central planner's program is:

$$
\max \min U(c, X)
$$

under the technical constraints (6) and (7), and the initial conditions (8).

We also assume Cobb-Douglas preferences.

Optimality conditions are still (15), (16) and (17).

It is useful to introduce the shadow price $q_{A}$ of technical progress $A$ in terms of the consumption good ${ }^{8}$, as well as the shadow value $Q_{A}=q_{A} A$ of the technical progress stock.

By definition, they satisfy

$$
\begin{gathered}
\dot{q}_{A}=(r-\gamma) q_{A}-F_{A} \\
\dot{Q}_{A}=r Q_{A}-F_{A} A
\end{gathered}
$$

where $r=F_{K}$ is the real interest rate.

\footnotetext{
${ }^{8}$ Arrow et al. [2003] provide a similar analysis in the case of a varying population rather than technical progress.
} 
A more concrete interpretation of these variables may be given. The technological stock $A$ may be considered as human capital and $q_{A}$ as the value of one unit of human capital. $F_{A}$ is the wage measured in efficiency units and $F_{A} A=(1-\alpha-\beta) Y$ the wage bill. The return to holding a unit of human capital is the sum of the wage $F_{A}$, the value $q_{A} \gamma$ of the exogenous increase in human capital and the capital gain $\dot{q}_{A}$. This return has to be equal to the real interest rate $r$. Lastly, human wealth $Q_{A}$ is the discounted value of future wages.

\subsection{Hartwick's rule}

An extended version of Hartwick's rule holds (Appendix 5):

$$
\dot{K}+q \dot{X}+q_{A} \dot{A}=0,
$$

or

$$
\dot{K}=q x-\gamma Q_{A}
$$

With technical progress, it is necessary to invest less than the rents coming from the extraction of the natural resource to maintain utility constant. The increase in human capital must be considered as a part of genuine saving.

We have, using the expressions of marginal productivities,

$$
\dot{Q}_{A}=\alpha \frac{Y}{K} Q_{A}-(1-\alpha-\beta) Y .
$$

Hartwick's rule (52) can be written as:

$$
\dot{K}=\beta Y-\gamma Q_{A}
$$

The equation of evolution of the resource stock (7) is:

$$
\dot{X}=-Y^{\frac{1}{\beta}} K^{-\frac{\alpha}{\beta}} A^{-\frac{1-\alpha-\beta}{\beta}} .
$$

Finally, the equation of accumulation of capital (6) and equation (54) give, with the expression of utility,

$$
Y=\frac{u X^{-\varepsilon}-\gamma Q_{A}}{1-\beta}
$$

We get a non-autonomous dynamical system in $Q_{A}, K$ and $X$ (equations (53), (54) and (55) where $Y$ is replaced by its expression (56)), for a given level of $u$. As we shall see below, this level will be determined through the constraint on the total utilization of the resource. 
Contrary to the case without technical progress, using Hartwick's rule does not simplify per se the resolution of the dynamical system. It simply leads to the substitution of variable $Q_{A}$ to $q$ and does not reduce the order of the dynamical system. It does provide a neat interpretation of the model however. Moreover it will lead us, in the case without resource amenity, to the Cairns and Long [2006] condition which will indeed reduce the dimension of the system.

\subsection{The long run evolution}

It is possible to transform system (53)-(55) in an autonomous system. To this end, we consider technical progress as augmenting the resource stock, and diminishing the capital stock at appropriate rates.

Let $J$ and $S$ be capital and the resource stock expressed in efficiency units, and $s$ the corresponding rate of resource utilization. Technical progress is considered as augmenting the resource with an elasticity $m_{X}$, diminishing the capital stock with an elasticity $m_{K}$ and augmenting the product with an elasticity $m_{A}$. These three elasticities are positive.

Thus we let:

$$
\begin{gathered}
Y=\left(K A^{-m_{K}}\right)^{\alpha}\left(x A^{m_{X}}\right)^{\beta} A^{m_{A}}, \\
J=K A^{-m_{K}}, \quad S=X A^{m_{X}}, \quad s=x A^{m_{X}} .
\end{gathered}
$$

On the long run path, $J, S$ and $s$ will be constant, which means that $K$ increases at rate $m_{K} \gamma$ while $X$ decreases at rate $m_{X} \gamma$. This justifies the assumptions about the opposite effects of technical progress on the two factors.

To be consistent with the production function, the three elasticities have to satisfy the following equation:

$$
-m_{K} \alpha+m_{X} \beta+m_{A}=1-\alpha-\beta .
$$

We obviously need $K$ and $Y$ to grow at the same rate in the long run, which requires

$$
m_{K}=m_{A} .
$$

Lastly, from equation (54), $Q_{A}$ must grow in the long run at the same rate as $K$ and $Y$, and equation (56) then imposes

$$
\varepsilon m_{X}=m_{K}
$$

in order to allow for a constant $X^{-\varepsilon} / Q_{A}$.

These conditions yield

$$
m_{X}=m \stackrel{\text { def }}{=} \frac{1-\alpha-\beta}{\beta+\varepsilon(1-\alpha)}, \quad m_{K}=m_{A}=\varepsilon m .
$$


We show in appendix 4, equation (73) that the long run values of the two capital stocks $J$ and $S$ satisfy the following equation

$$
J^{\alpha-1} S^{\beta}=B^{*}(m \gamma)^{1-\beta}
$$

where $B^{*}$ is a constant which does not depend on $u$. This determines an upward sloping curve in the $(J, S)$ space, which is the locus of potential stationary points. The particular stationary point $\left(J^{*}, S^{*}\right)$ which will be reached can only be determined through the analysis of the dynamical system and the determination of the highest possible utility level for which the resource constraint is satisfied.

We thus have characterized the long run path of the economy. In the long run, the deflated values $S$ and $J$ of the resource and capital stocks remain constant. Capital, production and consumption grow at the constant rate $\varepsilon m \gamma$ while the resource stock decreases at rate $m \gamma$. Technical progress now allows the marginal productivity of capital to remain constant even if resource use decreases and capital accumulates.

Even with technical progress, the amenity value of the resource does not prevent its complete depletion in the long run, when the utility is CobbDouglas. Yet the long run rate of depletion $m \gamma$ is a decreasing function of $\varepsilon$. The higher the amenity value, the lower the long run rate of depletion is. On the other hand, the physical rate of growth $\varepsilon m \gamma$ is an increasing function of $\varepsilon$. The will to slow down resource depletion thus induces the economy to implement faster physical growth. This may appear paradoxical but derives from the will to maintain a constant level of utility. This level is $u=c X^{\varepsilon}$. A higher taste for the amenity of the resource leads to a slower decrease of the resource stock but to faster decrease of its contribution to utility. The only way to compensate is to implement faster physical growth. Growth now appears as a way to compensate for resource depletion while maintaining utility. Thanks to capital accumulation, growth does not mean faster resource depletion.

\subsection{The case without resource amenity}

Let us now look at the case without resource amenity, when $\varepsilon=0$ and $m=(1-\alpha-\beta) / \beta$.

$J$ is identical to $K$ whereas $X=S A^{-m}$, while $B^{*}=1 / \alpha$.

There is no physical growth in the long run, as the objective is simply to maintain consumption constant. Physical capital converges to a constant level. The economy relies solely on technical progress to compensate for the reduction of resource use. 
The stationary point is such that

$$
K=\frac{\alpha}{m \gamma} c, \quad S=\alpha^{-\frac{\alpha}{\beta}}(m \gamma)^{\frac{\alpha-\beta}{\beta}} c^{\frac{1-\alpha}{\beta}}, \quad Q_{A}=\frac{\beta}{\gamma} c
$$

The dynamical model is (see the system (67)-(69) in appendix 5):

$$
\begin{gathered}
\dot{Q}_{A}=\left(\alpha \frac{Q_{A}}{K}-(1-\alpha-\beta)\right) Y \\
\dot{K}=\beta Y-\gamma Q_{A} \\
\dot{S}=-Y^{\frac{1}{\beta}} K^{-\frac{\alpha}{\beta}}+m \gamma S
\end{gathered}
$$

with :

$$
Y=\frac{c-\gamma Q_{A}}{1-\beta}
$$

The sustained level of consumption $c$ and the initial value of technical progress $Q_{A 0}$ are two unknowns to be determined.

The resolution can be simplified by using a relationship derived by Cairns and Long [2006], which allows us to drop one of the dynamic equations while introducing a condition linking the two unknowns $c$ and $Q_{A 0}$. Unfortunately, this method doesn't apply in the general case.

With our notations, Cairns and Long [2006] prove that the non-explosive solutions of the system satisfy the following equation (see Appendix 5):

$$
\frac{Q_{A}}{1-\alpha-\beta}=\frac{q X}{\beta} \text {. }
$$

Thus the values of the technological and natural stocks remain proportional to their share in production, along the optimal path.

Using (15) and the expression of the production function condition, (65) becomes

$$
Q_{A}=(1-\alpha-\beta) Y^{-(1-\beta) / \beta} K^{\alpha / \beta} S .
$$

Together with (64), this allows us to express $c$ as a function of $K, S, Q_{A}$. This relation holds at any time and in particular at time zero, so that we get a relationship between $c$ and $Q_{A 0}$, say

$$
c=c^{f}\left(K_{0}, S_{0}, Q_{A 0}\right),
$$

which supplements the system (61)-(64)). It is easy to check that this system, for a given $c$, has a saddle-point structure. The problem reduces to finding 
the convergent arm of a two-dimensional saddle point, taking into account the relationship between $c$ and $Q_{A 0}$.

Figure 3 describes the converging arm of the saddle-point for a given consumption level $c$. If we start from a relatively low level $K_{0}$ of capital, in comparison with initial resource stock $X_{0}$, the optimal path is to accumulate capital, while the shadow value $Q_{A}$ of the technology and the production level increase. The capital stock tends to a constant.

Figure 4 describes the trajectory of capital $K$ and the deflated resource stock $S$ when $c$ and $Q_{A 0}$ take their optimal values. The set of possible long run situations is the curve with positive slope. Starting from given initial stocks, the economy chooses the highest possible sustainable level of consumption permitting to attain one of the stationary points. This is equivalent to exhausting the resource in the long run: $S$ tends to a positive constant, but the undeflated resource stock $X$ tends to zero. A higher level of $c$ would exhaust the resource in finite time while a lower level would not exhaust the resource but would be suboptimal. This figure makes precise the discussion sketched in Cairns and Long [2006].

Figure 4 is drawn for the following calibration:

$$
\begin{gathered}
\alpha=.4 \quad \beta=.2 \quad \gamma=.01 \\
K_{0}=10 \quad X_{0}=10
\end{gathered}
$$

The long run equilibrium is

$$
K^{*}=39.8 \quad X^{*}=0.99
$$

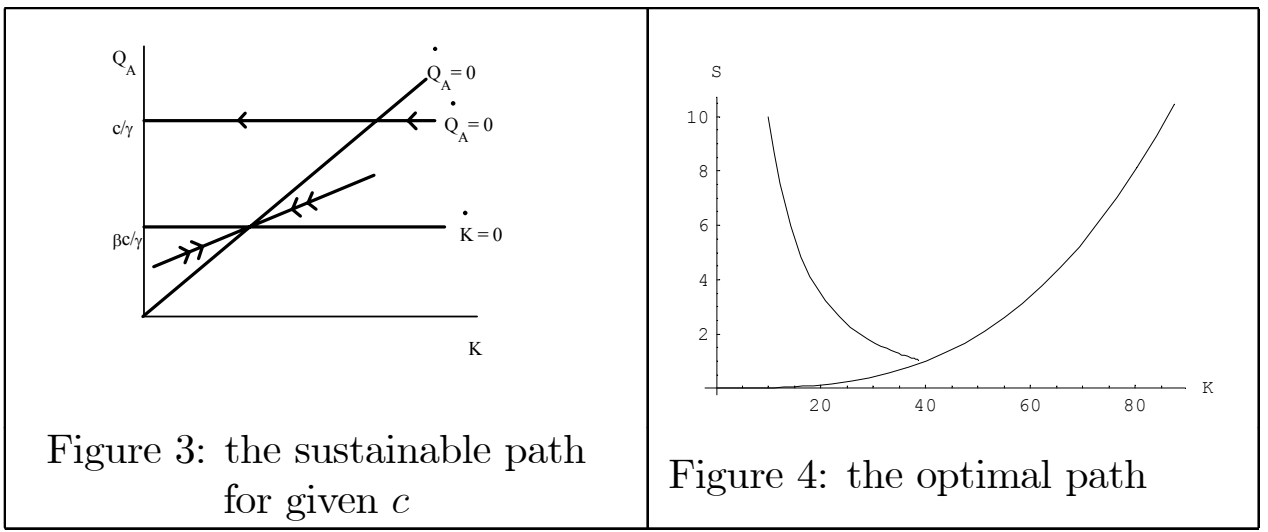

\subsection{The case with resource amenity}

In this case, we have a three dimensional system, with two unknowns $Q_{A 0}$ and $u$. This model can be solved numerically. 
We retain the previous calibration and now assume $\varepsilon=0.1$.

The following diagrams (figure 5) describe the evolutions and compare them to those of the case without resource amenity. They describe undeflated variables.
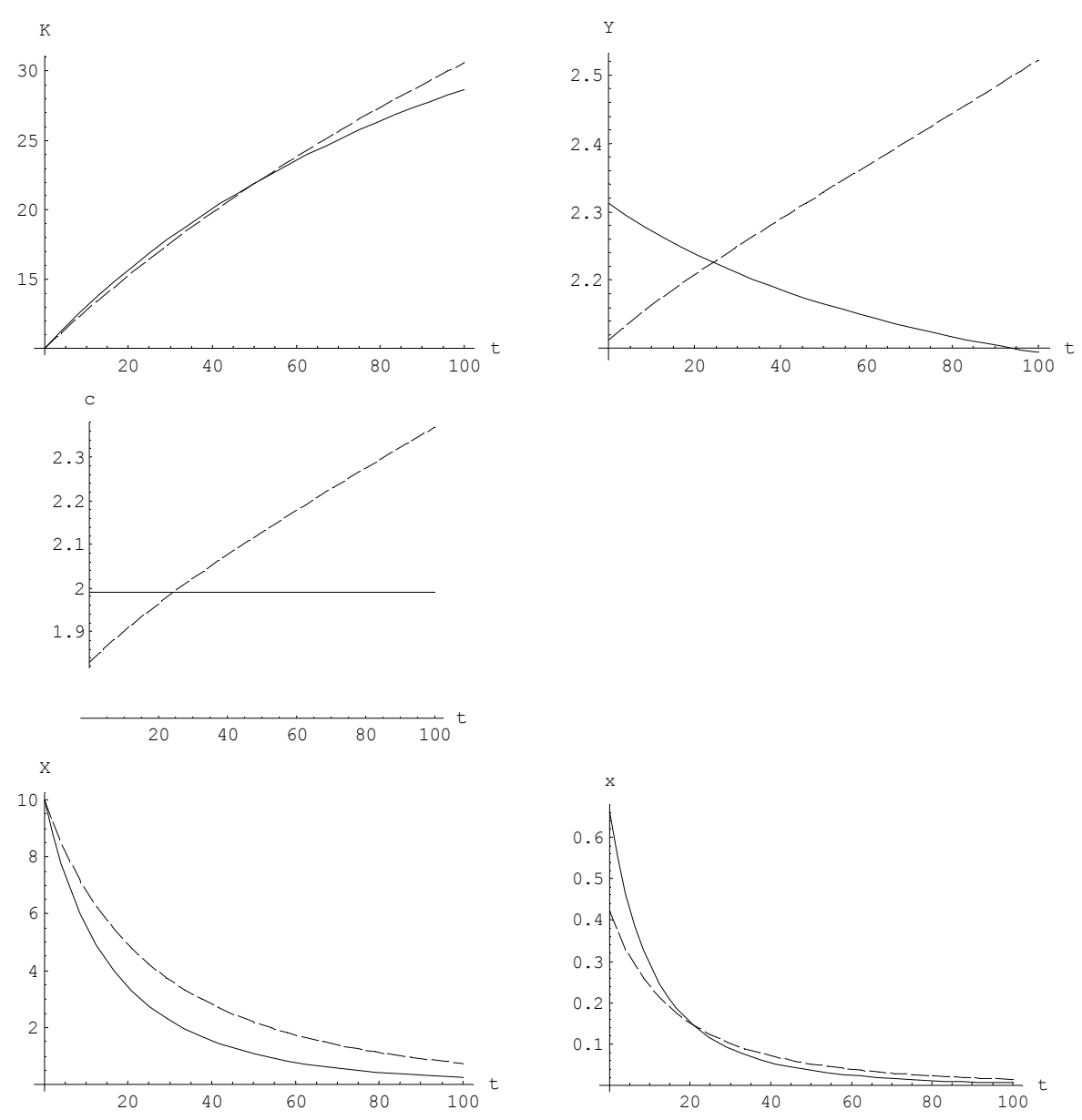

Figure 5: Comparaison of the maximin paths without (solid line) and with (dashed line) resource amenity, in the case of technical progress

In the case without resource amenity, agents maintain a constant level of consumption. Investment and technical progress both compensate for the unavoidable reduction in the use of natural resources. As technical progress builds up, investment may slow down. With constant consumption, this is compatible with a reduction of production. Eventually, production and the capital stock stabilize to constant levels and technological progress becomes sufficient to compensate for the decrease in resource use. 
In the case with resource amenity, consumption must increase in order to compensate for the unavoidable decrease in resource amenity. As the initial resource stock is high, a low initial level of consumption is sufficient to attain the sustainable utility level. Since they care about the size of the resource stock, agents economize on resource use. They consume, produce and invest less than in the case without resource amenity, which allows slower depletion of the resource stock. As this stock unavoidably decreases, the economy must increase its reliance on physical production. Capital accumulation, with the help of technological progress, allows the economy to maintain a high rate of growth, without depleting too quickly the resource stock. As could be expected, the resource stock remains for ever higher than in the case of no resource amenity.

\section{Conclusion}

Hartwick's rule appears as a simple and useful rule governing the equitable use of resources and the compensating saving and investment effort required to preserve the well-being of future generations. We have shown that it applies in a wide range of situations, when the resource stock has an amenity value for consumers as well as when technical progress affects intergenerational trade-offs.

The use of simple models, with Cobb-Douglas or CES utility, enabled us to provide explicit or quasi-explicit characterizations of optimal equitable paths and to describe how capital accumulation and increased consumption may or may not compensate for the unavoidable decrease of the natural capital stock.

Further research should be able to extend our results in various directions. Hartwick's rule covers the case of renewable resources. The main lines of the analysis are preserved although it seems difficult to get explicit solutions in this framework. The same is true for non-Cobb-Douglas technologies.

Introducing endogenous technical change would provide a better description of the ways man-made investment could compensate for the depletion of natural resources. This is undoubtedly a large and important research avenue. Exogenous technical progress appeared however as a useful and somewhat abstract framework to examine how economic progress will, presumably, help society to handle the depletion of natural resources and how intergenerational trade-offs should reflect both this depletion and the perspective of higher productivity. 


\section{References}

[2003] Arrow, K. J., P. Dasgupta and K.-G. Mäler, 2003, The genuine savings criterion and the value of population, Economic Theory, 20, 217-225.

[2007] Asheim, G. B., W. Buchholz; J. M. Hartwick, T. Mitra and C. Withagen, 2007, Constant savings rates and quasi-arithmetic population growth under exhaustible resource constraints, Journal of Environmental Economics and Management, 53, 213-229.

[2003] Asheim, G. B., W. Buchholz and C. Withagen, 2003, The Hartwick rule: myths and facts, Environmental and Resource Economics, 25, 129-150.

[2005] Buchholz, W., Dasgupta, S. and T. Mitra, 2005, Intertemporal equity and Hartwick's rule in an exhaustible resource model, Scandinavian Journal of Economics, 107, 547-561.

[2006] Cairns, R. D. and N. V. Long, 2006, Maximin: a direct approach to sustainability, Environment and Development Economics, 11, 275300 .

[1974] Dasgupta, P. and G. Heal, 1974, The optimal depletion of exhaustible resources, Review of Economic Studies (symposium), 3-28.

[1980] Dixit, A., Hammond, P. and M. Hoel, 1980, On Hartwick's rule for regular maximin paths of capital accumulation and resource depletion, Review of Economic Studies, 47, 551-556.

[1999] Hamilton, K. and M. Clemens, 1999, Genuine saving in developing countries, World Bank Economic Review, 13, 33-56.

[2007] Hamilton, K. and C. Withagen, 2007, Savings growth and the path of utility, Canadian Journal of Economics, 40, 703-713.

[1977] Hartwick, J., 1977, Intergenerational equity and the investing of rents from exhaustible resources, American Economic Review, 66(5), 972974 .

[1985] Krautkraemer J.A. (1985), Optimal growth, resource amenities and the preservation of natural environments, Review of Economic Studies, 52, 153-170.

[1992] Leonard, D. and N. V. Long, 1992, Optimal Control Theory and Static Optimization in Economics, Cambridge University Press, Cambridge. 
[1972] Rawls, J., 1972, A Theory of Justice, Clarendon Press, Oxford.

[1974] Solow, R., 1974, Intergenerational equity and exhaustible resources, Review of Economic Studies (symposium), 29-45.

[1998] Stollery, K. R., 1998, Constant utility paths and irreversible global warming, Canadian Journal of Economics, 31(3), 730-742.

[1998] Withagen, C. and G. B. Asheim, 1998, Characterizing sustainability: The converse of Hartwick's rule, Journal of Economic Dynamics and Control, 23, 159-165.

[2006] Withagen, C., Asheim, G. B. and W. Buchholz, 2003, On the sustainable program in Solow's model, Natural Resources Modeling, 16, $219-21$.

\section{Appendix 1: Proof that the utilitarist objec- tive function tends to maximin as $\sigma$ tends to zero}

We consider the utilitarist objective function

$$
\hat{W}=\left[\rho \int_{0}^{\infty} e^{-\rho t} u_{t}^{1-1 / \sigma} d t\right]^{\sigma /(\sigma-1)}
$$

where $\rho>0$ is an arbitrary utility discount rate and $\sigma>0$ the intertemporal elasticity of substitution.

Assume that $u_{t}$ is a continuous function of time defined on $(0, \infty)$, which tends to finite limits when $t$ tends to zero or infinity. Let $\underline{u}$ be the lower bound of $u_{t}$ on $(0, \infty)$ (it is either the minimum of $u_{t}$ or one of the limits). Assume $\underline{u}>0$.

To simplify notations, let $\kappa=\sigma /(1-\sigma)$. As $\sigma$ will tend to zero, we assume it to be smaller than one, so that $\kappa$ is positive and will tend to zero.

Let

$$
\hat{W}(\kappa)=\left[\rho \int_{0}^{\infty} e^{-\rho t} u_{t}^{-1 / \kappa} d t\right]^{-\kappa} .
$$

We have

$$
\hat{W}(\kappa)=\underline{u}\left[\rho \int_{0}^{\infty} e^{-\rho t}\left(\frac{u_{t}}{\underline{u}}\right)^{-1 / \kappa} d t\right]^{-\kappa} .
$$


As $u_{t} / \underline{u} \geq 1,\left(u_{t} / \underline{u}\right)^{-1 / \kappa} \leq 1$, which implies

$$
\rho \int_{0}^{\infty} e^{-\rho t}\left(\frac{u_{t}}{\underline{u}}\right)^{-1 / \kappa} d t \leq \rho \int_{0}^{\infty} e^{-\rho t} d t=1
$$

and therefore $\hat{W}(\kappa) \geq \underline{u}, \forall \kappa$, which implies

$$
\lim \inf _{\kappa \rightarrow 0} \hat{W}(\kappa) \geq \underline{u} .
$$

This basically reflects the fact that the objective functional is increasing in $u_{t}$.

For $\varepsilon>0$, let

$$
I_{\varepsilon}=\left\{t \in(0, \infty): u_{t} / \underline{u} \leq 1+\varepsilon\right\}
$$

and

$$
\mu(\varepsilon)=\rho \int_{I_{\varepsilon}} e^{-\rho t} d t
$$

As $u_{t}$ is continuous and $\underline{u}$ is the lower bound of $u_{t}, \mu(\varepsilon)>0, \forall \varepsilon>0$.

Let $J_{\varepsilon}$ be the complement of $I_{\varepsilon}$ in $(0, \infty)$;

$$
\begin{gathered}
\rho \int_{0}^{\infty} e^{-\rho t}\left(\frac{u_{t}}{\underline{u}}\right)^{-1 / \kappa} d t=\rho \int_{I_{\varepsilon}} e^{-\rho t}\left(\frac{u_{t}}{\underline{u}}\right)^{-1 / \kappa} d t+\rho \int_{J_{\varepsilon}} e^{-\rho t}\left(\frac{u_{t}}{\underline{u}}\right)^{-1 / \kappa} d t \\
\geq \rho \int_{I_{\varepsilon}} e^{-\rho t}\left(\frac{u_{t}}{\underline{u}}\right)^{-1 / \kappa} d t \geq \rho \int_{I_{\varepsilon}} e^{-\rho t}(1+\varepsilon)^{-1 / \kappa} d t=(1+\varepsilon)^{-1 / \kappa} \mu(\varepsilon)
\end{gathered}
$$

where the last inequality follows from the fact that $t \in I_{\varepsilon}$ implies $\left(u_{t} / \underline{u}\right)^{-1 / \kappa} \geq$ $(1+\varepsilon)^{-1 / \kappa}$.

Thus

$\hat{W}(\kappa)=\underline{u}\left(\rho \int_{0}^{\infty} e^{-\rho t}\left(\frac{u_{t}}{\underline{u}}\right)^{-1 / \kappa} d t\right)^{-\kappa} \leq \underline{u}\left((1+\varepsilon)^{-1 / \kappa} \mu(\varepsilon)\right)^{-\kappa}=\underline{u}(1+\varepsilon) \mu(\varepsilon)^{-\kappa}$.

As $\mu(\varepsilon)^{-\kappa}$ tends to one as $\kappa$ tends to zero,

$$
\lim \sup _{\kappa \rightarrow 0} \hat{W}(\kappa) \leq \underline{u}(1+\varepsilon), \quad \forall \varepsilon>0 .
$$

This implies

$$
\lim \sup _{\kappa \rightarrow 0} \hat{W}(\kappa) \leq \underline{u}
$$

and therefore

$$
\lim _{\kappa \rightarrow 0} \hat{W}(\kappa)=\underline{u} .
$$


Thus the usual property that the CES function tends to the min function holds in this continuous time setting.

Define now

$$
W=\int_{0}^{\infty} e^{-\rho t} \frac{u_{t}^{1-1 / \sigma}}{1-1 / \sigma} d t=-\frac{\sigma}{1-\sigma} \hat{W}^{-\frac{1-\sigma}{\sigma}}
$$

Maximizing $\hat{W}$ and $W$ are equivalent, since $W$ is a monotonically increasing function of $\hat{W}$.

\section{Appendix 2: The shadow price of equity}

We solve the model using the method of Léonard and Long [1992] but make explicit the shadow prices of equity put forward by Cairns and Long [2006]. As suggested in Léonard and Long [1992], we treat $u$ as a state variable, rather than a control parameter, and avoid the technical difficulties of the Cairns and Long's method.

The central planner seeks to maximize

$$
\int_{0}^{\infty} \rho e^{-\rho t} u d t
$$

under the constraints

$$
\begin{gathered}
\dot{K}_{t}=F\left(K_{t}, x_{t}\right)-c_{t}, \\
\dot{X}_{t}=-x_{t}, \\
\dot{u}=0, \\
u\left(c_{t}, X_{t}\right) \geq u,
\end{gathered}
$$

and the initial conditions

$$
K_{0} \text { and } X_{0} \text { given. }
$$

The lagrangian is:

$$
\mathcal{L}=\rho u+\lambda_{t}\left[F\left(K_{t}, x_{t}\right)-c_{t}\right]-\mu_{t} x_{t}+\psi_{t} .0+\theta_{t}\left[u\left(c_{t}, X_{t}\right)-u\right],
$$

and the first order necessary conditions can be written, with $q_{t}=\mu_{t} / \lambda_{t}$ the 
shadow price of the resource stock in terms of capital,

$$
\begin{aligned}
\theta_{t} u_{c}\left(c_{t}, X_{t}\right) & =\lambda_{t} \\
\frac{\dot{\lambda}_{t}}{\lambda_{t}} & =\rho-F_{K} \\
F_{x} & =q_{t} \\
\frac{\dot{q}_{t}}{q_{t}} & =F_{K}-\frac{u_{X} / u_{c}}{q} \\
\dot{\psi}_{t} & =\rho \psi_{t}-\rho+\theta_{t} .
\end{aligned}
$$

As the state variable $u$ is free at both endpoints, we have $\psi_{0}=0$ and $\lim _{\infty} e^{-\rho t} \psi_{t}=0$, and therefore

$$
\int_{0}^{\infty} e^{-\rho t}\left(\theta_{t}-\rho\right) d t=0
$$

i.e.

$$
\int_{0}^{\infty} e^{-\rho t} \theta_{t} d t=1
$$

The shadow value of equity is the discounted shadow price of the last constraint $u\left(c_{t}, X_{t}\right) \geq u, w_{t}=e^{-\rho t} \theta_{t}$. Using the first order conditions, it can be written as:

$$
w_{t}=e^{-\rho t} \theta_{t}=e^{-\rho t} \frac{\lambda_{t}}{u_{c}\left(c_{t}, X_{t}\right)}=e^{-R_{t}} \frac{\lambda_{0}}{u_{c}\left(c_{t}, X_{t}\right)},
$$

with $R_{t}=\int_{0}^{t} F_{K}\left(K_{s}, x_{s}\right) d s$. It is independent of $\rho$.

We check easily that the maximin path is solution of the problem

$$
\max \int_{0}^{\infty} w_{t} u\left(c_{t}, X_{t}\right) d t
$$

under the technical constraints and the initial conditions.

Thus this shadow price plays the role of a discount factor.

\section{Appendix 3: The Dixit-Hammond-Hoel rule}

In order to follow their method, it is useful to introduce the price $p$ of the amenity agents derive from holding the resource stock. Total consumption will then appear as $c+p X$, and optimality conditions (15) and (16) may be written as

$$
\begin{aligned}
F_{x} & =q \\
\dot{q}+p & =r q,
\end{aligned}
$$


with

$$
\begin{aligned}
r & =F_{K}, \\
p & =u_{X} / u_{c} .
\end{aligned}
$$

Then differentiate with respect to time the equations of accumulation of capital (6) and the resource stock (7) to get

$$
\ddot{K}=r \dot{K}+q \dot{x}-\dot{c}=r \dot{K}-q \ddot{X}-\dot{c}
$$

i.e., using the optimality conditions above,

$$
\ddot{K}+q \ddot{X}=r \dot{K}-\dot{c}=r \dot{K}-\dot{c}+(r q-\dot{q}-p) \dot{X}
$$

or

$$
\ddot{K}+q \ddot{X}+\dot{q} \dot{X}-r(\dot{K}+q \dot{X})=-\dot{c}-p \dot{X} .
$$

Define total investment, or genuine saving, as

$$
G=\dot{K}+q \dot{X}
$$

and note that

$$
\dot{u}=u_{c} \dot{c}+u_{X} \dot{X}=u_{c}(\dot{c}+p \dot{X})
$$

to obtain

$$
\dot{G}-r G=-\dot{u} / u_{c} .
$$

A competitive constant utility path is characterized by $\dot{G}-r G=0$, that is

$$
e^{-R} G=e^{-R}(\dot{K}+q \dot{X})=\text { constant. }
$$

It remains to prove that $G=0$, i.e. the converse of Hartwick's rule. The proof follows Buchholz et al. [2005], who study the case where utility only depends on consumption.

With a Cobb-Douglas production function, we have

$$
x=\beta \frac{Y}{q}=\beta \frac{\dot{K}+c}{q}=\beta \frac{G+q x+c}{q}>\beta \frac{G}{q} .
$$

Moreover,

$$
\begin{aligned}
G & =e^{R} G_{0} \\
q & =e^{R}\left(q_{0}-\int_{0}^{t} e^{-R_{s}} p_{s} d s\right)<e^{R} q_{0}
\end{aligned}
$$


Then, if $G_{0}>0, G / q>G_{0} / q_{0}$ and

$$
x>\beta \frac{G_{0}}{q_{0}} .
$$

The right-hand side member is a positive constant. Then the resource constraint $\int_{0}^{\infty} x d t<X_{0}$ cannot be satisfied. The case $G_{0}<0$ can be immediately ruled out, as it implies that capital accumulation does not compensate for resource depletion; then consumption collapses and utility cannot be maintained constant. We deduce that $G_{0}=G=0 \forall t$. The converse of Hartwick's rule holds.

\section{Appendix 4: Resolution of the Cobb-Douglas}

\section{case}

From equations (23), (25) we have

$$
\dot{K}=\beta \frac{C(u, X)}{1-\beta}=\beta \frac{u X^{-\varepsilon}}{1-\beta},
$$

and, using (34) and (35),

$$
\dot{K}=\beta\left(\frac{\alpha-\beta}{\phi} B\right)^{\beta /(1-\beta)}\left(B K^{-(\alpha-\beta) / \beta}\right)^{-\varepsilon / \phi} .
$$

and, eventually,

$$
\dot{K}=A K^{\pi}
$$

with

$$
\pi=\frac{\varepsilon(\alpha-\beta)}{\beta \phi}, \quad A=\beta\left(\frac{\alpha-\beta}{\phi}\right)^{\frac{\beta}{1-\beta}} B^{\frac{\beta}{(1-\beta) \phi}},
$$

where we use the fact that $\phi=[\beta+(1-\beta) \varepsilon] / \beta$.

We can write

$$
\pi=\frac{\varepsilon(\alpha-\beta)}{\beta+(1-\beta) \varepsilon}=1-\frac{\beta+(1-\alpha) \varepsilon}{\beta+(1-\beta) \varepsilon}
$$

As $\alpha>\beta, \pi$ is positive and smaller than one.

The preceeding differential equation can be integrated. It yields:

$$
K=\left[(1-\pi) A t+K_{0}^{1-\pi}\right]^{\frac{1}{1-\pi}},
$$


from which we deduce

$$
X=B^{1 / \phi} K^{-\frac{\alpha-\beta}{\beta \phi}}=B^{1 / \phi}\left[(1-\pi) A t+K_{0}^{1-\pi}\right]^{-\frac{\alpha-\beta}{\beta+\varepsilon(1-\alpha)}} .
$$

Finally, the shadow value of equity (Cairns and Long [2006]), defined in Appendix 2, is

$$
w_{t}=e^{-R_{t}} \frac{\lambda_{0}}{u_{c}}
$$

We have

$$
\begin{aligned}
\frac{\dot{w}}{w} & =-F_{K}-\frac{\dot{u}_{c}}{u_{c}}=-F_{K}-\varepsilon \frac{\dot{X}}{X}=-\alpha \frac{Y}{K}-\varepsilon \frac{\dot{X}}{X}=-\frac{\alpha}{\beta} \frac{\dot{K}}{K}-\varepsilon \frac{\dot{X}}{X} \\
& =-\frac{\alpha}{\beta} A K^{\pi-1}+\varepsilon \frac{\alpha-\beta}{\beta\left(1+\frac{1-\beta}{\beta} \varepsilon\right)} A K_{t}^{\pi-1}=\left(-\frac{\alpha}{\beta}+\pi\right) A K^{\pi-1} \\
& =-\frac{\left(\frac{\alpha}{\beta}-\pi\right) A}{(1-\pi) A t+K_{0}^{1-\pi}} .
\end{aligned}
$$

This equation can be integrated:

$$
w=w_{0}\left(\frac{K_{0}^{1-\pi}}{(1-\pi) A t+K_{0}^{1-\pi}}\right)^{\frac{\frac{\alpha}{\beta}-\pi}{1-\pi}} .
$$

$w_{0}$ is obtained by using $\int_{0}^{\infty} w d t=1$ :

$$
\int_{0}^{\infty} w d t=w_{0} K_{0}^{\frac{\alpha}{\beta}-\pi} \int_{0}^{\infty}\left((1-\pi) A t+K_{0}^{1-\pi}\right)^{-\frac{\alpha}{\beta}-\pi} \frac{w_{0} K_{0}^{1-\pi}}{A\left(\frac{\alpha}{\beta}-1\right)}
$$

and

$$
\int_{0}^{\infty} w d t=1 \Leftrightarrow w_{0}=\frac{A\left(\frac{\alpha}{\beta}-1\right)}{K_{0}^{1-\pi}}
$$

As

$$
\frac{\alpha}{\beta}-\pi=\frac{\alpha+\varepsilon(1-\alpha)}{\beta+\varepsilon(1-\beta)}, \quad \frac{\frac{\alpha}{\beta}-\pi}{1-\pi}=\frac{\alpha+\varepsilon(1-\alpha)}{\beta+\varepsilon(1-\alpha)}
$$

we get the formula (39) in the text. 


\section{Appendix 5: The case of technical progress}

\section{Hartwick's rule}

Optimality conditions for problem (48) are (15), (16) and (49):

$$
\begin{aligned}
F_{x} & =q, \\
\dot{q}+p & =r q, \\
\dot{q}_{A} & =(r-\gamma) q_{A}-F_{A} .
\end{aligned}
$$

Derive with respect to time the equations of accumulation of capital and the resource stock to get

$$
\ddot{K}=r \dot{K}+q \dot{x}+F_{A} \dot{A}-\dot{c}=r \dot{K}-q \ddot{X}+F_{A} \dot{A}-\dot{c}
$$

i.e., using the optimality conditions below,

$\ddot{K}=r \dot{K}-q \ddot{X}+F_{A} \dot{A}-\dot{c}=r \dot{K}-q \ddot{X}+F_{A} \dot{A}-\dot{c}+(r q-\dot{q}-p) \dot{X}+\left((r-\gamma) q_{A}-\dot{q}_{A}-F_{A}\right) \dot{A}$ or, with $\dot{c}=\frac{\dot{u}}{u_{c}}-p \dot{X}$,

$$
\ddot{K}=r \dot{K}-q \ddot{X}-\frac{\dot{u}}{u_{c}}+(r q-\dot{q}) \dot{X}+\left((r-\gamma) q_{A}-\dot{q}_{A}\right) \dot{A}
$$

i.e.

$$
\ddot{K}+q \ddot{X}+\dot{q} \dot{X}+q_{A} \ddot{A}+\dot{q}_{A} \dot{A}=r\left(\dot{K}+q \dot{X}+q_{A} \dot{A}\right)-\frac{\dot{u}}{u_{c}}
$$

i.e.

$$
\frac{d}{d t}\left(\dot{K}+q \dot{X}+q_{A} \dot{A}\right)=r\left(\dot{K}+q \dot{X}+q_{A} \dot{A}\right)-\frac{\dot{u}}{u_{c}} .
$$

Genuine saving is in this case

$$
G=\dot{K}+q \dot{X}+q_{A} \dot{A},
$$

and the Hartwick's rule is

$$
\dot{K}+q \dot{X}+q_{A} \dot{A}=0 .
$$

\subsection{The deflated dynamical system}

We have $J=K A^{-\varepsilon m}, S=X A^{m}, s=x A^{m}$. Let $\tilde{Y}=J^{\alpha} s^{\beta}=Y A^{-\varepsilon m}$, $\widetilde{Q}_{A}=Q_{A} A^{-\varepsilon m}$ and $\tilde{c}=c A^{-\varepsilon m}$ be the deflated values of production, technical progress and consumption. The utility level is $u=c X^{\varepsilon}=\tilde{c} S^{\varepsilon}$. The transformed dynamical system (53)-(56) is then

$$
\frac{\tilde{\widetilde{Q}}_{A}}{\widetilde{Q}_{A}}=\alpha \frac{\widetilde{Y}}{J}-(1-\alpha-\beta) \frac{\widetilde{Y}}{\widetilde{Q}_{A}}-\varepsilon m \gamma,
$$




$$
\begin{gathered}
\frac{\dot{J}}{J}=\beta \frac{\widetilde{Y}}{J}-\gamma \frac{\widetilde{Q}_{A}}{J}-\varepsilon m \gamma, \\
\frac{\dot{S}}{S}=-\frac{\widetilde{Y}^{\frac{1}{\beta}} J^{-\frac{\alpha}{\beta}}}{S}+m \gamma,
\end{gathered}
$$

with

$$
\widetilde{Y}=\frac{u S^{-\varepsilon}-\gamma \widetilde{Q}_{A}}{1-\beta} .
$$

Let $\psi=\frac{\widetilde{Y}}{J}$ be the productivity of capital. It is easy to show that the stationary state of the transformed dynamical system (67)-(70) is unique.

From equations (67) and (68), the stationary value of $\psi$ is a solution of the following equation:

$$
g(\psi)=\alpha \beta \psi^{2}-(\beta+\varepsilon(1+\beta)) \gamma m \psi+(\varepsilon \gamma m)^{2}=0 .
$$

It is obviously of the form

$$
\psi^{*}=B^{*} m \gamma
$$

with a coefficient $B^{*}$ solution of equation $g(B)=\alpha \beta B^{2}-(\beta+\varepsilon(1+\beta)) B+$ $\varepsilon^{2}=0$. $>$ From (68), $\psi^{*}$ has to be larger than $\varepsilon \gamma m / \beta$, as $\tilde{Q}_{A} / J$ must be positive, and therefore $B^{*}$ larger than $\varepsilon / \beta$. As $g(0)>0$ and $g(\varepsilon / \beta)=$ $-\left((1-\alpha) \varepsilon^{2}+\beta \varepsilon\right) / \beta<0$ the equation has two roots, the larger of which is the solution.

Then

$$
\begin{gathered}
\frac{\widetilde{Q}_{A}}{J}=\left(\beta B^{*}-\varepsilon\right) m, \\
J^{\alpha-1} S^{\beta}=B^{*}(m \gamma)^{1-\beta}, \\
\frac{u S^{-\varepsilon}}{J}=\left(B^{*}-\varepsilon\right) \gamma m .
\end{gathered}
$$

\section{The Cairns and Long relation}

In the case $\varepsilon=0$, let us define the new variable

$$
H=\left(\frac{Q_{A}}{q}-\frac{1-\alpha-\beta}{\beta} X\right) A^{m} .
$$


We have

$$
\begin{aligned}
\dot{H} & =\left(\frac{\dot{Q}_{A}}{q}-\frac{\dot{q}}{q} \frac{Q_{A}}{q}-\frac{1-\alpha-\beta}{\beta} \dot{X}\right) A^{m}+m \gamma H \\
& =\left(\frac{r Q_{A}-(1-\alpha-\beta) Y}{q}-r \frac{Q_{A}}{q}+\frac{1-\alpha-\beta}{\beta} x\right) A^{m}+m \gamma H \\
& =m \gamma H
\end{aligned}
$$

as

$$
q=\beta \frac{Y}{x} .
$$

The non-explosion of $H$ requires $H=0$ that is

$$
Q_{A}=\frac{1-\alpha-\beta}{\beta} q X
$$

which is Cairns and Long [2006] relationship. 